\title{
Efficacy and Safety Study of CA330 Hemadsorption Device on IL-6 Removal in Septic Patients: Study Protocol of a Multicenter Randomized Controlled Trial
}

\author{
Yao Nie*, Zhiyi Jiang, Zimeng Liu, Xiangdong Guan \\ Department of Critical Care Medicine, The First Affiliated Hospital of Sun Yat-sen University, Guangzhou, China \\ Email: ^nieyao@mail.sysu.edu.cn
}

How to cite this paper: Nie, Y., Jiang, Z.Y., Liu, Z.M. and Guan, X.D. (2021) Efficacy and Safety Study of CA330 Hemadsorption Device on IL-6 Removal in Septic Patients: Study Protocol of a Multicenter Randomized Controlled Trial. Open Journal of Epidemiology, 11, 517-524.

https://doi.org/10.4236/ojepi.2021.114041

Received: October 31, 2021

Accepted: November 27, 2021

Published: November 30, 2021

Copyright $\odot 2021$ by author(s) and Scientific Research Publishing Inc. This work is licensed under the Creative Commons Attribution International License (CC BY 4.0).

http://creativecommons.org/licenses/by/4.0/

\begin{abstract}
Background: Sepsis persists to be the leading cause of morbidity and mortality worldwide with the huge cost of health care resources. Besides adequate antibiotics and infectious source control, definitive therapy is still being studied. The activation of multiple pro- and anti-inflammatory mediators plays a key role in the sepsis process. The application of adsorption may help deactivate and decrease the peak elevation of these mediators in the earlier course of sepsis, when levels of endotoxins and cytokines are extremely high. However, the clinical evidence to support hemadsorption for removing endotoxins and/or pro-inflammatory mediators in sepsis remains incompetent and controversial. In this study protocol, we aimed to test the efficacy of removing cytokines and the safety of a new hemadsorption device, CA330, in septic patients. Design: This is a multicenter randomized controlled clinical trial enrolling 8 tertiary hospitals in China. A total of 144 patients will be randomly divided into the experimental group and the control group according to the ratio of 1:1. The primary endpoint is the reduction rate of IL-6 serum concentration between the initiation of the first adsorption and end with the second adsorption. Discussion: To our knowledge, this clinical trial is the first to evaluate the efficacy and safety of the CA330 hemadsorption device in sepsis patients. Our study will raise the level of evidence for the treatment of sepsis patients with hemadsorption.
\end{abstract}

\section{Keywords}

Sepsis, Hemadsorption, CA330, Inflammatory Mediators, IL-6 


\section{Background}

Sepsis is one of the leading causes of morbidity and mortality worldwide. Moreover, the magnitude of health care resources utilized to manage septic patients is huge. All these severe facts call for constant efforts to optimize therapy. At present, conventional treatment of sepsis typically starts with resuscitative measures. However, the only definitive therapy is adequate antibiotics and infectious source control. According to the latest international consensus, sepsis is defined as life-threatening organ dysfunction caused by a dysregulated host response to infection [1]. Fortunately, research has led to a better understanding of the pathophysiology of sepsis, in which the activation of multiple pro- and anti-inflammatory mediators plays a key role [2]. Understanding of this complex mechanism has led to the development of treatment strategies aimed at restoring a balanced immune response by eliminating/deactivating these inflammatory mediators. Whilst animal models of sepsis have provided encouraging results with strategies aiming at immune response modulation; clinical studies in patients using targeted pharmacological approaches have so far proved disappointing.

Besides AKI, Renal Replacement Therapy (RRT) is applied to remove inflammatory mediators extracorporeally. Across the different modalities, the application of adsorption may help deactivate and decrease the peak elevation of these mediators in the earlier course of sepsis, when levels of endotoxins and cytokines are extremely high [3]. Recently, attempts to improve the outcome of sepsis patients with such devices, i.e., CytoSorb cytokine hemadsorption and Polymyxin B (Toraymyxin) endotoxin adsorption, have seen a certain renaissance. The evidence for CytoSorb (CS) is limited to case reports/series and a few RCTs, but it is growing. It was recently studied in a multicenter RCT in 100 mechanically ventilated patients with severe sepsis or septic shock and acute lung injury or ARDS [4]. Though IL-6 was removed, hemadsorption did not lead to lower plasma IL-6-levels. Significant differences in the multiple organ dysfunction scores, ventilation time and time course of oxygenation were not detected either. Toraymyxin was developed in Japan in the early 1990s. Though two multicenter trials demonstrated that Toraymyxin may improve hemodynamics and organ dysfunction [5], even reduce 28-day mortality [6], two subsequent larger clinical studies were negative [7] [8]. HA330, an extracorporeal hemoperfusion device that uses neutral microporous resin, also showed effectiveness in sepsis treatment. Despite the two RCTs were small, HA330 decreased inflammatory mediators, along with an improvement in hemodynamics, respiratory parameters, mortality, and ICU length of stay [9] [10]. Overall, the clinical evidence to date supporting hemadsorption for removing endotoxins and/or pro-inflammatory mediators in sepsis remains incompetent and controversial.

CA330 (Jafron Biomedical Co., Ltd., Zhuhai, China) is a hemadsorption device containing hemocompatible, porous polymeric beads capable of removing cytokines and other mid-molecular weight toxins from the blood by size exclusion and surface adsorption. Compared with HA330, improved resin synthesis 
technology makes CA330 a better performance in removing cytokines. This trial is the first to evaluate CA330 efficacy of cytokine reduction using the change in plasma interleukin (IL)-6 concentrations over time as a primary outcome. Although the trial was neither designed nor powered to evaluate the outcome, we also evaluated organ function parameters as well as 28 -day all-cause mortality.

\section{Method}

\subsection{Trial Design and Setting}

This is a multicenter randomized controlled clinical trial enrolling 8 tertiary hospitals in China. Study participants will not be enrolled until the study is approved by the institutional ethics boards for all study sites. All enrolled patients or legally authorized representatives will provide written informed consent.

\subsection{Participants}

Patients are eligible to participate if they meet the following criteria:

1) Hospitalized patients aged 18 - 75 years, regardless of gender;

2) Meet the clinical criteria of sepsis according to the Third International Consensus Definitions for Sepsis [1];

3) The diagnosis of sepsis is established within last 48 hours.

4) Written informed consent is signed, and agreement to participate in all visits, examinations and treatments as required by the research program is achieved. If a patient is not able to give consent, the legal representative is asked to consent.

Patients are excluded if there are following exclusion criteria:

1) End-stage renal disease that need maintenance hemodialysis treatment;

2) Congenital or acquired immunodeficiency disorders or those who had received organ transplantation within one year;

3) Prescription of immunosuppressive medications (tripterygium wilfordii, mycophenolate mofetil, cyclophosphamide, FK506, etc.) or prednisolone for more than $10 \mathrm{mg} /$ day (or the same dose of other hormone therapy) within 30 days;

4) Coagulation dysfunction, severe bleeding tendency (PT, APTT prolonged significantly with bleeding, or fibrinogen less than $1.2 \mathrm{~g} / \mathrm{L}$ with bleeding), active bleeding or uncontrolled acute massive bleeding within 24 hours;

5) Malignant tumors, consumptive diseases, site of infection cannot be cleared (ie surgical patients who cannot be operated on) or body weight less than $35 \mathrm{~kg}$;

6) A terminal state of organ failure (end stage of COPD, pulmonary heart disease, heart dysfunction stage-IV, brain death or persistent vegetative state, chronic liver disease with hepatic encephalopathy, coagulation dysfunction, fluid retention and hepatocellular jaundice);

7) Following results are detected during screening: platelet count $<50 * 10^{9} / \mathrm{L}$, neutrophil count $<0.5 * 10^{9} / \mathrm{L}$, hemoglobin $<70 \mathrm{~g} / \mathrm{L}$;

8) Despite of adequate volume resuscitation, vasopressors and hormones, the mean arterial blood cannot maintain above $65 \mathrm{mmHg}$; 
9) Those who have been participating in or participated in another interventional clinical study within 30 days prior to enrollment, such as the clinical study of related drugs or medical devices affecting immunity (i.e., Xuebijing, ulinastatin, CRRT, etc.);

10) Anaphylaxis to materials of cardiopulmonary bypass, anticoagulants and hemoperfusion or other serious allergic history;

11) Those who are not suitable for this clinical trial, which is determined by researchers, such as pregnant or lactating women, current drug addicts, patients with severe mental or neurological disorders, and those who have a history of alcohol abuse and cannot be terminated.

\subsection{Treatment Allocation and Randomization}

Eligible patients are stratified by site and randomly assigned in a 1:1 ratio to either CA330 hemoperfusion plus conventional medical therapy group or conventional medical therapy group. The randomization is performed by researchers on the central randomization system, provided by the Department of Biostatistics, Southern Medical University. In this study, the center coded random number table is produced by stratified and sectional randomization method. SAS 9.4 statistical software is used to generate random coding tables with serial numbers (001-144) according to the number of cases allocated in each center and the proportion of experimental group and control group (1:1). The length of selected sections (block) and random seed number are sealed together as confidential data. The random number table is provided by the statisticians of the Department of Biostatistics, Southern Medical University.

\subsection{Study Interventions}

The experimental group receives routine treatment of sepsis combined with cytokine adsorption column (CA330) perfusion, and the control group receives routine treatment of sepsis only.

Hemoperfusion treatments are performed using a perfusion machine via centrally inserted standard dialysis catheters at a prescribed blood flow rate of 100 $300 \mathrm{~mL} / \mathrm{min}$. In the beginning, lower flow rate is recommended, if there is no discomfort, and then gradually increases. Each patient received 2 hemoperfusion treatments within 24 hours with a target duration for each treatment of 120 - 180 minutes (minimum of 120 minutes). The shorter the interval between the two hemoperfusion, the better. It is suggested that the second hemoperfusion be performed within 0 - 5 hours after the first one.

Anticoagulation was recommended with low-molecular-weight heparin in arterial line of the circuit at the dose of $60-80 \mathrm{IU} / \mathrm{kg}$. No additional dose is required. The activity of anticoagulant factor $\alpha$ can be monitored at 60 minutes. It is suggested that the activity of anticoagulant factor a should be maintained at $500-1000 \mathrm{U} / \mathrm{L}$ in subjects without bleeding tendency and $200-400 \mathrm{U} / \mathrm{L}$ in subjects with bleeding tendency. If the clinical condition is limited, it is not manda- 
tory to monitor this parameter.

Since there is no recommendation of timing to start RRT in sepsis, we take KDIGO Clinical Practice Guideline for Acute Kidney Injury into account [11]. Emergency RRT therapy includes life-threatening hyperkalemia, acidosis, pulmonary edema and uremia complications; non-emergency RRT treatment includes solute control, fluid clearance, acid-base imbalance correction, volume control, nutritional support, drug delivery and maintenance of water and electrolyte balance. RRT blood flow is set to $100-250 \mathrm{ml} / \mathrm{min}$. For patients with unstable hemodynamics, the blood flow rate was gradually increased from 50 $100 \mathrm{ml} / \mathrm{min}$. While for patients with stable hemodynamics, the blood flow rate is set to about $200 \mathrm{ml} / \mathrm{min}$. The prescription dose of RRT is $25-30 \mathrm{ml} / \mathrm{kg} / \mathrm{h}$ to achieve the actual therapeutic dose of $20-25 \mathrm{ml} / \mathrm{kg} / \mathrm{h}$. For RRT, the preferred hemofilter model is AV600S. For hospitals without this type of hemofilter, M100 is the alternative offer. The proportion and length of RRT are taken as secondary result when analyze. The anticoagulant regimen of RRT is not mandatory. It is suggested to select the anticoagulant regimen according to the sequence of "lowmolecular-weight heparin, unfractionated heparin, citric acid". The unfractionated heparin is given at 0/30/60/90 minutes to maintain ACT at $180-240$ seconds.

All sites agree, when feasible, to follow the tenets of the Surviving Sepsis Campaign clinical practice guidelines for management of sepsis [12].

\subsection{Sample Size Estimation}

According to the previous studies, the average decline rate of IL- 6 in experimental group is $10 \%$ lower than that of the control group. The overall standard deviation of the two groups is difficult to estimate. We assume that the effect is $20 \%$. Using nQuery Advisor + nTerim 4.0 software, 64 cases in each group are estimated to provide a bilateral test level of $0.05 \%$ and $80 \%$ power. Considering $10 \%$ drop-off rate, the final numbers of cases in each group are determined to be 72 cases, a total of 144 cases are required. 144 subjects were randomly divided into the experimental group and the control group according to the ratio of $1: 1$.

\subsection{Endpoint}

The primary endpoint of the study is the reduction rate of IL-6 serum concentration at the initiation of first adsorption and at the end of the second adsorption.

Secondary endpoints are the reduction rate of IL-10, TNF- $\alpha$, lactic acid serum concentration at the initiation of first adsorption and at the end of the second adsorption, the dynamic change of APACHE II score, ventilation time, oxygenation index, urinary output, length of vasopressors, catecholamine index, RRT incidency and length of treatment, ICU and hospital length of stay, and 28-day all-cause mortality. Data are collected at the following time points: screening, hemadsorption period, 1 day \pm 2 hours after the end of second adsorption, 3 day \pm 4 hours after the end of second adsorption, $7 \pm 2$ days after the end of second 
adsorption, $14 \pm 3$ days after the end of second adsorption, $28 \pm 4$ days after the end of second adsorption.

The safety parameters include vital signs (including body temperature, heart rate, respiratory rate and blood pressure), $\mathrm{CBC}$, coagulation function, liver function (ALT, AST, TBIL, DBIL, total protein, albumin), renal function (creatinine, blood urea), electrolyte (potassium, sodium, calcium, phosphorus, magnesium) before and after the hemadsorption. Adverse effects are also recorded.

\subsection{Statistical Analysis}

Statistical analyses are performed using SAS (SAS Institute Inc) software for Windows version 9.4. Descriptive analyses are performed on baseline variables using mean (SD), median (interquartile range [IQR]), or proportions and percentages as appropriate. Measurement data are tested by two independent sample size $t$ test or corrected $t$ test (when variance heterogeneity is assumed), or the corresponding non-parametric method. Categorical data are tested by Pearson Chi-square test. Rank data are analyzed by rank sum test.

The primary endpoint intergroup comparison is conducted using covariance analysis (ANCOVA) with baseline as covariate, taking into account the central effect and possible interaction effects. With the validation of superiority, if the lower limit of $95 \%$ confidence interval between the experimental group and the control group is greater than 0 , it can be inferred that the experimental group is better than the control group.

The secondary endpoints intra-group comparisons are conducted by paired t-test or Wilcoxon sign rank test for quantitative variables, and paired chi-square test for qualitative variables. For intergroup comparisons, quantitative indicators are tested by two independent samples $\mathrm{t}$ test or Wilcoxon rank sum test, and qualitative indicators are tested by chi-square test. Grade factors are tested by Wilcoxon rank sum test.

\section{Discussion}

The latest guideline for the management of sepsis and septic shock didn't support renal replacement therapy without definitive indications for renal replacement therapy, including uremic complications, refractory academia, refractory fluid overload or hyperkalemia [13]. However, the updated recommendations were based on new RCTs including septic patients with AKI and far-fetched results favoring delayed RRT due to central venous access device infections. Besides, the guideline only mentioned continuous and intermittent renal replacement therapy, but hemadsorption is a distinct modality with special mechanisms and treatment goals. A literature review showed that CytoSorb adsorber had beneficial effects on survival rate and inflammatory responses of sepsis both in animal experiments and patients [14]. To our knowledge, this clinical trial is the first to evaluate the efficacy and safety of the CA330 hemadsorption device in sepsis patients. Our study will raise the level of evidence for the treatment of sepsis pa- 
tients with hemadsorption.

\section{Conflicts of Interest}

The authors declare no conflicts of interest regarding the publication of this paper.

\section{References}

[1] Singer, M., Deutschman, C.S., Seymour, C.W., et al. (2016) The Third International Consensus Definitions for Sepsis and Septic Shock (Sepsis-3). The Journal of the American Medical Association, 315, 801-810. https://doi.org/10.1001/jama.2016.0287

[2] Gotts, J.E. and Matthay, M.A. (2016) Sepsis: Pathophysiology and Clinical Management. British Medical Journal, 353, i1585. https://doi.org/10.1136/bmj.i1585

[3] Ankawi, G., Neri, M., Zhang, J., Breglia, A., Ricci, Z. and Ronco, C. (2018) Extracorporeal Techniques for the Treatment of Critically Ill Patients with Sepsis beyond Conventional Blood Purification Therapy: The Promises and the Pitfalls. Critical Care, 22, Article No. 262. https://doi.org/10.1186/s13054-018-2181-Z

[4] Schädler, D., Pausch, C., Heise, D., et al. (2017) The Effect of a Novel Extracorporeal Cytokine Hemadsorption Device on IL-6 Elimination in Septic Patients: A Randomized Controlled Trial. PLoS ONE, 12, e0187015.

https://doi.org/10.1371/journal.pone.0187015

[5] Vincent, J., Laterre, P., Cohen, J., Burchardi, H., Bruining, H., Lerma, F., et al. (2005) A Pilot-Controlled Study of a Polymyxin B-Immobilized Hemoperfusion Cartridge in Patients with Severe Sepsis Secondary to Intra-Abdominal Infection. Shock, 23, 400-405. https://doi.org/10.1097/01.shk.0000159930.87737.8a

[6] Cruz, D.N., Antonelli, M., Fumagalli, R., Foltran, F., Brienza, N., Donati, A., et al. (2009) Early Use of Polymyxin B Hemoperfusion in Abdominal Septic Shock: The EUPHAS Randomized Controlled Trial. The Journal of the American Medical Association, 301, 2445-2452. https://doi.org/10.1001/jama.2009.856

[7] Payen, D.M., Guilhot, J., Launey, Y., et al. (2015) Early Use of Polymyxin B Hemoperfusion in Patients with Septic Shock Due to Peritonitis: A Multicenter Randomized Control Trial. Intensive Care Medicine, 41, 975-984.

https://doi.org/10.1007/s00134-015-3751-Z

[8] Dellinger, R.P., Bagshaw, S.M., Antonelli, M., et al. (2018) Effect of Targeted Polymyxin B Hemoperfusion on 28-Day Mortality in Patients with Septic Shock and Elevated Endotoxin Level: The EUPHRATES Randomized Clinical Trial. The Journal of the American Medical Association, 320, 1455-1463. https://doi.org/10.1001/jama.2018.14618

[9] Huang, Z, Wang, S.-R., Su, W. and Liu, J.-Y. (2010) Removal of Humoral Mediators and the Effect on the Survival of Septic Patients by Hemoperfusion with Neutral Microporous Resin Column. Therapeutic Apheresis and Dialysis, 14, 596-602. https://doi.org/10.1111/j.1744-9987.2010.00825.x

[10] Huang, Z., Wang, S.-R., Yang, Z.-L. and Liu, J.-Y. (2013) Effect on Extrapulmonary Sepsis-Induced Acute Lung Injury by Hemoperfusion with Neutral Microporous Resin Column. Therapeutic Apheresis and Dialysis, 17, 454-461. https://doi.org/10.1111/j.1744-9987.2012.01083.x

[11] Khwaja, A. (2012) KDIGO Clinical Practice Guidelines for Acute Kidney Injury. Nephron Clinical Practice, 120, c179-c184. https://doi.org/10.1159/000339789 
[12] Rhodes, A., Evans, L.E., Alhazzani, W., et al. (2017) Surviving Sepsis Campaign: International Guidelines for Management of Sepsis and Septic Shock: 2016. Intensive Care Medicine, 43, 304-377. https://doi.org/10.1007/s00134-017-4683-6

[13] Evans, L., Rhodes, A., Alhazzani, W., et al. (2021) Surviving Sepsis Campaign: International Guidelines for Management of Sepsis and Septic Shock 2021. Intensive Care Medicine, 47, 1181-1247. https://doi.org/10.1007/s00134-021-06506-y

[14] Houschyar, K.S., Pyles, M.N., Rein, S., Nietzschmann, I., Duscher, D., Maan, Z.N., Weissenberg, K., Phillipps, H.M., Strauss, C., Reichelt, B. and Siemers, F. (2017) Continuous Hemadsorption with a Cytokine Adsorber during Sepsis-A Review of the Literature. The International Journal of Artificial Organs, 40, 205-211.

https://doi.org/10.5301/ijao.5000591 